\title{
Médiévales
}

Langues, Textes, Histoire

\section{De la copie à l'édition : Francesc Argilagues et les manuscrits médicaux aux premiers temps de l'imprimerie (fin $\mathrm{xv}^{\mathrm{e}}$-début $\mathrm{xvI}^{\mathrm{e}}$ siècle)}

Traduit de l'espagnol par Nicolas Weill-Parot

Jon Arrizabalaga

\section{(2) OpenEdition}

Journals

Édition électronique

URL : https://journals.openedition.org/medievales/2483

DOI : $10.4000 /$ medievales. 2483

ISSN : $1777-5892$

Éditeur

Presses universitaires de Vincennes

Édition imprimée

Date de publication : 1 juin 2007

Pagination : 119-134

ISBN : 978-2-84292-202-3

ISSN : 0751-2708

Référence électronique

Jon Arrizabalaga, « De la copie à l'édition : Francesc Argilagues et les manuscrits médicaux aux premiers temps de l'imprimerie (fin XVe-début XVIe siècle) », Médiévales [En ligne], 52 I printemps 2007, mis en ligne le 06 septembre 2009, consulté le 24 avril 2022. URL : http://

journals.openedition.org/medievales/2483; DOI : https://doi.org/10.4000/medievales.2483

Tous droits réservés 


\section{DE LA COPIE À L'ÉDITION : \\ Francesc Argilagues et les manuscrits médicaux aux premiers temps de l'imprimerie (fin $\mathrm{XV}^{\mathrm{e}}$-début $\mathrm{XVI}^{\mathrm{e}}$ siècle)}

Voilà plusieurs années, à l'occasion d'un travail sur les étudiants de médecine de la Couronne d'Aragon dans les universités de Sienne, Pise, Ferrare et Padoue durant la période $1470-1520^{1}$, mon attention fut particulièrement attirée par l'un de ces étudiants espagnols. Il apparaissait sous le nom de «Franciscus Ioannis Arguelagues de Valentia» dans la prosopographie monumentale d'Armando F. Verde sur l'Université de Pise à la fin du $\mathrm{Xv}^{\mathrm{e}}$ siècle ${ }^{2}$, et avait été le propriétaire d'un manuscrit latin autographe conservé à la Bibliothèque Vaticane (ms. Rossianus 672). Le contenu de ce codex, de caractère médical, avait été partiellement décrit par Paul Oskar Kristeller et par le même Verde ${ }^{3}$. La consultation du recensement des éditions de la Renaissance des œuvres de Galien par Richard J. Durling ${ }^{4}$, motivée par une étude sur l'Articella dans les débuts de l'imprimerie ( $c a$ 1476-1534) ${ }^{5}$, me fit rencontrer un «Franciscus Argilagues » dans le rôle d'éditeur de l'Articella

1. J. Arrizabalaga, L. García Ballester, F. Salmón, «A propósito de las relaciones intelectuales entre la Corona de Aragón e Italia (1470-1520) : Los estudiantes de medicina valencianos en los estudios generales de Siena, Pisa, Ferrara y Padua », Dynamis, 9, 1999, p. 117-147.

2. A. F. VERDE, Lo Studio fiorentino, 1473-1503. Ricerche e Documenti, Florence/Pistoia, 1973, vol. II, p. 670-671 ; vol. III/1, p. 269-270, 342 ; vol. IV/1, p. 270-271.

3. P. O. KRISTEller, Iter Italicum. A finding list of uncatalogued or incompletely catalogued Humanistic manuscripts of the Renaissance in Italian and other libraries, Londres/Leyde, 1977-1992, 6 vol., vol. II, p. 470 ; VERDE, op. cit., vol. IV/1, p. 271.

4. R. J. Durling, «A chronological census of Renaissance editions and translations of Galen », Journal of the Warburg and Courtauld Institutes, 24, 1961, p. 230-305.

5. Sur l'Articella - une collection d'écrits médicaux à but didactique très diffusée au Moyen Âge latin, à laquelle je me référerai plus amplement tout le long de ce travail - à l'époque de l'imprimerie, on peut voir J. Arrizabalaga, «The Death of a Medical Text: The Articella and the Early Press », dans R. French, J. Arrizabalaga, A. Cunningham et L. García Ballester éd., Medicine from the Black Death to the French Disease, Aldershot, 1998, p. 184-220; J. Arrizabalaga, The Articella in the early press, ca 1476-1534, Cambridge/Barcelone, 1998. 
imprimée à Venise en 1483. En m'aidant de divers catalogues de bibliothèques $\mathrm{j}$ 'ai pu avoir la confirmation du fait qu'il s'agissait du même individu, et découvrir qu'il s'était également occupé d'une autre édition imprimée du Quattrocento de l'Articella (Venise 1487). Le répertoire encyclopédique des humanistes de Mario E. Cosenza ${ }^{6}$ me mit ensuite sur la piste d'un autre fruit de l'activité éditoriale d'Argilagues : trois éditions imprimées du Conciliator de Pietro d'Abano $(\dagger 1316)$, toutes du $\mathrm{Xv}^{\mathrm{e}}$ siècle (Venise 1483, Pavie 1490 et Venise 1496).

De cet ensemble d'informations tirées de ces sources émergea un personnage dont la biographie permet non seulement d'illustrer, dans le milieu de la médecine universitaire, la période de transition entre la copie de manuscrits et les premières éditions imprimées, mais aussi de reconstruire les débuts du métier d'éditeur scientifique et les questions de nature technique ou idéologique auxquelles furent confrontés ceux qui cultivèrent cette nouvelle occupation surgie dans le sillage immédiat de l'invention de Gutenberg.

\section{Francesc Argilagues (fl. $c a$ 1470-1508) ${ }^{7}$}

Le floruit de Francesc Argilagues doit se situer entre 1470 environ, date où le Valencien commença ses études de médecine dans le studium de Sienne, et 1508, année au début de laquelle (le 20 janvier) il fit relier le manuscrit autographe déjà évoqué ${ }^{8}$. Bien que l'absence de répertoires exhaustifs des médecins valenciens des $\mathrm{XV}^{\mathrm{e}}$ et $\mathrm{XVI}^{\mathrm{e}}$ siècles nous empêche de l'affirmer de manière catégorique, les diverses informations relatives à Argilagues durant les presque quarante ans qui séparent ces deux dates induisent à penser que ce dernier, comme d'autres de ses contemporains valenciens formés également en Italie, choisit de s'installer là-bas après la fin de ses études. Nous ne connaissons pas les raisons que le poussèrent à faire cela et nous ignorons si, même occasionnellement, il retourna dans sa ville natale plus tard à un certain moment de sa vie. Quoi qu'il en soit, il semble clair que la répression sévère exercée par l'Inquisition à l'encontre de la bourgeoisie conversa de

6. M. CosENZA, Biographical and bibliographical dictionary of the Italian Humanists, 13001800, Boston, 1962-1967, 4 vol., vol. I, p. 258.

7. Sur la vie et l'œuvre d'Argilagues on peut voir J. Arrizabalaga, L. García Ballester et J. L. GIL ARISTU, « Del manuscrito al primitivo impreso : la labor editora de Francesc Argilagues (fl. ca 1470-1508) en el Renacimiento médico italiano », Asclepio, 43/1, 1991, p. 3-50. La quasitotalité des informations qui sont ici reproduites procède des données autobiographiques fournies par Argilagues lui-même dans le codex manuscrit autographe Rossianus 672 de la Bibliothèque Vaticane (désormais BAV), et de VERDE, op. cit.

8. BAV, Rossianus 672, fo $195 \mathrm{r}^{\circ}$ : " Finis. Laus Deo Januarií 201508 Veneciis per me Franciscum Argilagues de Valentia artium et medicina doctorem ». L'écriture au tracé moins ferme et à une colonne - en contraste avec le reste du codex écrit sur deux colonnes - que l'on trouve dans les trois derniers folios écrits de ce codex $\left(\mathrm{f}^{\mathrm{o}} 193 \mathrm{r}^{\circ}-195 \mathrm{r}^{\circ}\right)$ pourrait bien être le reflet de l'âge avancé d'Argilagues. 
Valence - que l'on se souvienne, entre autres, du cas de la famille de Juan Luis Vives et celui du médecin Lluís Alcanyís ( ca 1440-1506), accusés de cryptojudaïsme - dans les dernières années $\mathrm{du} \mathrm{Xv}^{\mathrm{e}}$ siècle et les premières du $\mathrm{XVI}^{\mathrm{e}}$ siècle, n'était guère propice au retour à la maison de ceux qui, comme Argilagues, avaient trouvé l'occasion de se créer une place professionnelle dans les terres italiennes où, de surcroît, ils pouvaient bénéficier d'une plus grande liberté intellectuelle et religieuse ${ }^{9}$.

À partir de l'année académique 1473-1474, Francesc Argilagues se transféra à Pise, université où il poursuivit sa formation jusqu'à l'obtention du doctorat en arts et médecine le 11 août 1477. Tout semble indiquer que son déplacement de Sienne à Pise, à l'instar de ses compagnons d'étude, les frères Jeroni et Gaspar Torrella, eux aussi Valenciens, fut lié au départ d'Alessandro Sermoneta, l'un de ses professeurs de médecine à Sienne, qui avait accepté la charge de l'une des deux leçons ordinaires de médecine théorique au studium qui venait d'être ouvert par Laurent de Médicis à Pise. Selon toute probabilité, le prestige et l'influence de Sermoneta sur ses élèves attirèrent à Pise également Argilagues.

Nous savons qu'Argilagues participa de façon très active à la vie estudiantine et que pendant l'avant-dernière année académique (1475-1476) il fut élu pour la dite « leçon festive», honneur que l'on avait coutume d'accorder aux étudiants les plus éminents. Pour cette leçon il reçut en septembre 1476 le salaire de 64 livres florentines. Habituellement, on obtenait le doctorat l'année suivante, et ce fut ce qui se passa justement pour Argilagues à la date déjà indiquée. Son certificat d'aptitude au grade de docteur fut accrédité par trois professeurs du studium, qui agirent comme promotori du candidat : Pierleone da Spoleto (mort en 1492), lecteur ordinaire de médecine pratique depuis l'année académique 1475-1476, le professeur de logique Giovanni da Venezia et Sermoneta lui-même ${ }^{10}$.

De toute évidence, les relations entre le disciple et son maître se prolongèrent après la fin des études médicales du premier. En effet, le 30 mars et le 14 avril 1479, Argilagues fut témoin de chacun des actes notariés établis à Pise sur les instances de Sermoneta, par lesquels ce dernier désignait des procurateurs, dans le premier cas, pour gérer en son absence les affaires qu'il avait à Sienne d'où il était originaire; et dans le second, pour accepter en son nom toute nomination par le gouvernement de Venise "pour enseigner la médecine et la philosophie au studium de Padoue». Le 15 juin 1479, Sermoneta fut nommé lecteur ordinaire de médecine théorique à l'Université de Padoue. Argilagues dut abandonner Pise à peu près au moment où Sermoneta

9. R. GARCía CÁRCEL, Orígenes de la inquisición española. El tribunal de Valencia, 14781530, Barcelone, 1976, p. 233-237. Sur Lluís Alcanyís, on peut voir Lluís AlCANYís, Regiment de la pestilència (València, [Nicolau Spindeler], ca 1490). Édition et étude par J. ARrizabalaga, Barcelone, sous presse.

10. Sur Pierleone da Spoleto, on peut voir M. Rotzoll, Pierleone da Spoleto : vita e opere di un medico del Rinascimento, Florence, 2001. 
accéda à sa nouvelle chaire. Peut-être alors suivit-il son maître et protecteur jusqu'à Padoue, où ce dernier demeura comme professeur jusqu'en 1484 ; ou bien peut-être se transféra-t-il directement dans la Venise voisine où il résidait dès 1483 - l'attestation la plus ancienne de sa présence dans cette ville est constituée par l'indication qu'il donne de l'année et du lieu de la reliure du codex - et où il était encore actif au début de l'année 1508, date à laquelle on perd définitivement sa trace. Entre les deux termes, sa présence dans cette ville semble attestée à différentes dates, où on le trouve se consacrant à l'exercice pratique de la médecine et, bien sûr, à ses activités éditoriales qui s'étendirent de 1483 à 1496. Au cours de ces quatorze années, Argilagues s'occupa de deux éditions imprimées de l'Articella (Venise 1483 et 1487) et de trois autres (Venise 1483 et 1496 ; Pavie 1490) du Conciliator de Pietro d'Abano. Au préalable, à l'époque où il était étudiant ou bien alors qu'il venait juste d'obtenir son doctorat (entre 1476 et 1479), Argilagues avait copié dans différentes villes de Toscane (Pise, Sienne, Florence, Pistoia) la plus grande partie des dix-huit textes médicaux que contient le codex Vaticanus Rossianus 672. Je consacrerai le reste de ce travail à l'examen assez minutieux des activités de notre médecin comme copiste et comme éditeur d'œuvres médicales.

\section{Argilagues, copiste d'œuvres médicales}

Dix des dix-huit traités médicaux inclus dans le codex autographe d'Argilagues appartiennent ou bien ont été attribués à Arnaud de Villeneuve : De vinis $\left(\mathrm{f}^{\circ} 5 \mathrm{r}^{\circ}-14 \mathrm{v}^{\circ}\right)$, Pronostica $\left(\mathrm{f}^{\circ} 34 \mathrm{r}^{\circ} \mathrm{b}-38 \mathrm{r}^{\circ} \mathrm{a}\right)$, De aquis medicinalibus $\left(\mathrm{f}^{\circ} 70 \mathrm{r}^{\circ} \mathrm{b}-74 \mathrm{r}^{\circ} \mathrm{a}\right)$, De dosi tiriacalium $\left(\mathrm{f}^{\mathrm{o}} 116 \mathrm{v}^{\circ} \mathrm{a}-120 \mathrm{v}^{\circ} \mathrm{a}\right)$, Experimenta $\left(\mathrm{f}^{\mathrm{o}} 137 \mathrm{r}^{\circ} \mathrm{a}-\right.$ $\left.144 \mathrm{r}^{\circ} \mathrm{a}\right)$, Quadam Arnaldi de Villanova $\left(\mathrm{f}^{\circ} 144 \mathrm{r}^{\circ} \mathrm{a}-146 \mathrm{r}^{\circ} \mathrm{b}\right)$, De considerationibus operis medicine ( $\left.\mathrm{f}^{\circ} 146 \mathrm{v}^{0} \mathrm{a}-177 \mathrm{v}^{\circ} \mathrm{b}\right)$, De humido radicali $\left(\mathrm{f}^{\mathrm{o}} 177 \mathrm{v}^{\circ} \mathrm{b}-188 \mathrm{v}^{0} \mathrm{a}\right)$ et le commentaire sur quelques Medicationis parabole $\left(\mathrm{f}^{\mathrm{o}} 188 \mathrm{v}^{\circ} \mathrm{b}-192 \mathrm{v}^{\circ}\right)^{11}$. Parmi les huit restants il en est deux, le Consilium de ardore urine $\left(\mathrm{f}^{\circ} 74 \mathrm{r}^{\circ} \mathrm{a}-\right.$ $\left.83 v^{\circ} \mathrm{b}\right)$ et le De urinis $\left(\mathrm{f}^{\mathrm{o}} 84 \mathrm{r}^{\mathrm{o}} \mathrm{a}-116 \mathrm{v}^{\circ} \mathrm{a}\right)$ de Pierlone da Spoleto ; la version latine, effectuée par le même Arnaud, du De medicinis simplicibus ( $\left(\mathrm{f}^{\mathrm{0}} 121 \mathrm{r}^{\mathrm{o}} \mathrm{a}-136 \mathrm{v}^{\circ} \mathrm{b}\right)$ d'Abu-1-Salt ; le traité De gradibus medicinarum d'al'Kindi (fo $\left.57 \mathrm{r}^{\circ} \mathrm{a}-63 \mathrm{v}^{\circ} \mathrm{a}\right)$; un antidotaire de tradition salernitaine ( $\left.\mathrm{f}^{\mathrm{o}} 64 \mathrm{r}^{\circ} \mathrm{a}-70 \mathrm{r}^{\circ} \mathrm{b}\right)$, le Dietarium $\left(\mathrm{f}^{\circ} 15 \mathrm{r}^{\circ}\right.$ $34 \mathrm{r}^{\circ} \mathrm{b}$ ) de Stephanus Arlandi ; et deux autres d'auteurs inconnus : De febribus $\left(38 \mathrm{r}^{\circ} \mathrm{b}-48 \mathrm{r}^{\circ} \mathrm{b}\right)$ et Experimenta et cure multarum egritudinum $\left(\mathrm{f}^{\circ} 48 \mathrm{r}^{\circ} \mathrm{b}-53 \mathrm{v}^{\circ} \mathrm{a}\right)$.

Dans quel but Argilagues a-t-il copié cet ensemble varié d'œuvres contenues dans ce codex ? Je ne peux hélas donner de réponse définitive à cette question, même si je peux, en revanche, avancer quelques hypothèses ${ }^{12}$. L'explication la plus immédiate de son activité de copiste est que celle-ci s'exer-

11. S. Giralt Soler, Arnau de Vilanova en la impremta renaixentista, Manresa, 2002, p. $28,168$.

12. BAV, Rossianus $672, \mathrm{f}^{\mathrm{o}} 116 \mathrm{v}^{\circ}$ : «Amen. Florencie scripta sunt 1478 lanuarii 18. Franciscus Argilagues ». 
çait uniquement pour son usage personnel, guidé par des intérêts médicaux théoriques et/ou pratiques. La présence des deux écrits cités de Pierleone da Spoleto, dont Argilagues acheva la copie à Florence le 18 janvier 1478, pourrait nourrir cette hypothèse. En fin de compte, Pierleone, qui était médecin personnel de Laurent de Médicis et l'un des membres les plus éminents du cercle néoplatonicien de Marsile Ficin, avait été professeur de médecine pratique d'Argilagues et l'un des promoteurs de son doctorat à l'Université de Pise $^{13}$. À son tour, Pierleone put agir comme moteur de l'évidente curiosité d'Argilagues pour l'œuvre d'Arnaud de Villeneuve. En effet, la bibliothèque personnelle de Pierleone comptait en abondance des œuvres médicales et spirituelles d'Arnaud, dont beaucoup avaient été copiées à l'époque de ses études à Paris ${ }^{14}$. Les deux faits pourraient être considérés comme 1'expression d'un regain d'intérêt pour les œuvres d'Arnaud et d'autres auteurs médicaux de la première scolastique (Bernard de Gordon, Pietro d'Abano, Taddeo Alderotti et Gentile da Foligno, entre autres) dans les cercles universitaires européens de l'époque ${ }^{15}$.

Mais l'activité de copiste d'Argilagues pourrait aussi répondre à des travaux de commande dont, pour une raison ou une autre, il garda une copie. Comme on le sait, la copie de manuscrits pour lesquels il existait une demande sur le marché constituait parfois une première occupation parmi les étudiants étrangers qui choisissaient une installation professionnelle en Italie au lieu d'un retour chez eux à l'issue de leurs études. Alessandro Sermoneta lui-même fut un employeur bien connu d'étudiants ultramontains dans ces occupations que le gradué de fraîche date Argilagues aurait acceptées dans le double dessein de remettre à flot ses économies qui risquaient à l'évidence d'être malmenées, et de maintenir la confiance de son protecteur ${ }^{16}$. Mais Argilagues pourrait aussi avoir travaillé comme copiste pour le fameux libraire florentin Vespasiano de Bisticci (1421-1488). Ce dernier disposait à Florence d'un scriptorium et d'un vaste réseau de copistes privés qui rassembla jusqu'à 45 secrétaires au moins, se consacrant tous à la préparation de manuscrits de toute œuvre qui leur était demandée par les Médicis et autre riches citoyens florentins ${ }^{17}$. L'existence d'une relation entre De Bisticci et Argilagues est suggérée par le fait qu'en juillet 1476, alors qu'il était encore un étudiant,

13. VERDE, op. cit., vol. II, p. 554-563.

14. Sur l'intérêt de Pierleone pour Arnaud de Villeneuve, voir L. García Ballester et E. Sanchez-SAlor éd., Arnaldi de Villanova opera medica omnia. XV. Commentum supra tractatum Galieni de malicia complexionis diverse, Barcelone, 1985, p. 142.

15. Sur le cas de l'Université de Salamanque, voir L. García BaLlester, « Galenismo y enseñanza médica en la Universidad de Salamanca del siglo XV », Dynamis, 21, 2001, p. 209247 à p. 244-251.

16. L. ZdeKauer, Lo studio di Siena nel Rinasciment, Milan, 1894, p. 89-93 ; T. PeSENTI, Professori e promotori di medicina nello studio di Padova dal 1405 al 1509, Trieste, 1984, p. 196-198.

17. Sur la figure et les activités de Vespasiano da Bisticci, voir G. M. CAGNI, Vespasiano da Bisticci e il suo epistolario, Rome, 1969, en particulier p. 46-85 ; R. HIRSCH, Printing, selling and reading, 1450-1550, $2^{\mathrm{e}}$ éd., Wiesbaden, 1974, p. 14. 
ce dernier désigna le premier ainsi qu'un autre commerçant florentin comme ses procurateurs auprès du studium de Pise pour s'occuper du recouvrement de la somme assignée à la lecture festive qui était à sa charge pendant l'année académique qui venait de s'achever ${ }^{18}$. Et, compte tenu de tout ce qui été dit, il ne paraît guère étonnant qu'Argilagues, durant les années où il résidait en Toscane, se soit vu confier la charge de copier les écrits d'Arnaud de Villeneuve, d'al-Kindi, et, qui plus est, de Pierleone de Spoleto - le médecin préféré de Laurent le Magnifique et, aux côtés d'Antonio Benivieni, de Bernardo Torni, de Marsile Ficin et d'autres encore, l'un des plus influents dans la culture florentine du dernier tiers du $\mathrm{Xv}^{\mathrm{e}}$ siècle ${ }^{19}$.

Enfin, on ne doit pas écarter non plus l'idée que certains de ces écrits aient été copiés dans le dessein de préparer des éditions imprimées de ces derniers, ce qui s'accorderait avec les activités éditoriales d'Argilagues qui étaient déjà bien développées. De fait, ce fut à partir de la décennie 1470 que la médecine et la philosophie naturelle universitaires commencèrent à passer par l'imprimerie, fondamentalement aux dépens des œuvres des autorités grecques (Hippocrate, Galien, Platon, Aristote, ...), arabes (Avicenne, Averroès, Mesue, Rhasès, ...) ou latines (Pline l'Ancien, Pietro d'Abano, Gentile da Foligno, Jacopo da Forli, ...) qui avaient joui d'une large circulation manuscrite durant les siècles médiévaux ${ }^{20}$; tandis que la présence d'imprimés d'auteurs contemporains (Gabrielle de Zerbi, Nicolò Leoniceno, Gaspar Torrella...) est à peine décelable avant la décennie $1490^{21}$. Si nous nous interrogeons sur la fortune imprimée des œuvres médicales que comprend le codex Rossianus, nous constatons que l'editio princeps des Opera d'Arnaud de Villeneuve ne devait pas paraître avant 1504, bien que depuis la décennie 1470 eussent été publiées de nombreuses œuvres particulières, séparées ou formant une partie de volumes de mélanges, parmi lequelles les suivantes, qui sont présentes dans le codex Rossianus : De humido radicali (Strasbourg, 1472-1474), De vinis

18. A. F. VERDE, op. cit., vol. III/1, p. 269.

19. K. PARK, Doctors and medicine in early Renaissance Florence, Princeton, 1985, p. 235236.

20. Parmi les jalons de ce processus on trouve les éditions imprimées de l'Historia naturalis de Pline l'Ancien (édition latine 1469), le Canon d'Avicenne (éd. lat. 1473), les Opera de Platon (éd. lat. 1484/1485 ; éd. grecque 1513), de Galien (éd. lat. 1490 ; éd. gr. 1525) et d'Aristote (éd. lat. 1482 ; éd. gr. 1495-97), et le Corpus Hippocraticum (éd. lat. 1525 ; éd. gr. 1526).

21. Pour une présentation assez exhaustive des premières éditions des œuvres de médecine et de philosophie naturelle, voir en les combinant A. C. KLEBS, Incunabula scientifica et medica [1938], Hildesheim, 1963 (reimpression en facsimile) ; M. B. STILlwell, The awakening interest in science during the first century of printing, 1450-1550. An annotated checklist of first editions viewed from the angle of their subject content. Astronomy - Mathematics - Medicine - Natural Science - Physics - Technology, New York, 1970. Sur les livres de médecine et de philosophie naturelle dans la première imprimerie, voir aussi L. GARCíA BALLESTER, «La nueva industria del libro médico y el renacer del humanismo médico latino », dans M. Fernández Álvarez et al., La cultura del renaixement. Homenatge al Pare Miquel Batllori, Bellaterra, 1993, p. 111128, à p. 120-121; W. EAMON, Science and the secrets of nature. Books of secrets in medieval and early modern culture, Princeton, 1994 ; J. PARDO TOMÁs, « La producción impresa de libros científicos en la Corona de Aragón (1475-1600) », dans E. SARASA et E. Serrano éd., La Corona de Aragón y el Mediterráneo (siglos XV y XVI), Zaragoza, 1995, p. 231-266. 
(Leipzig, ca 1500) et Medicationis parabole (Salamanque, 1501) ${ }^{22}$. Il est également intéressant de constater que le De gradibus medicinarum d'al' Kindi allait être publié en 1501 accompagné des commentaires d'Arnaud et des Parabole medicacionis de ce dernier ${ }^{23}$, et que l'editio princeps de deux petits traités de Pierleone de Spoleto ne verrait pas le jour avant 1514, lorsque Giorgio Arrivabene les imprima à Venise, aux frais des héritiers d'Ottaviano Scoto, à l'intérieur d'une collection d'écrits sémiologiques sur les pouls et les urines avec, en tête, ceux de Gilles de Corbeil ${ }^{24}$.

J'aimerais conclure cette section en formulant l'hypothèse selon laquelle Argilagues serait intervenu d'une certaine manière dans la préparation de la deuxième édition des Opera Arnaldi de Villanova (Venise, 19 janvier 1505). Cette édition a dû être préparée simultanément avec la première (Lyon, 18 janvier 1504) et inclut la même lettre dédicatoire de l'éditeur Thomas Murchius que celle- $\mathrm{ci}^{25}$. Cependant, certaines particularités de cette édition (ordre différent dans la disposition des œuvres, tabula des matières différente, notes dans les marges qui répètent in situ les entrées de la tabula) conduisent à penser à une intervention éditoriale d'une autre personne non identifiée ${ }^{26}$. Nous savons qu'Argilagues se trouvait à Venise au début de l'année 1505, grâce à une annotation de sa main qui se fait l'écho d'un incendie qui avait détruit le 25 janvier de cette année «tout l'édifice de la halle aux grains des Allemands ${ }^{27}$. Il parait également significatif que cette édition vénitienne des Opera Arnaldi ait été imprimée et financée par les mêmes artisans de l'édition plus tardive du Conciliator dont se chargea Argilagues (Venise, Boneto Locatelli pour Ottaviano Scotti, 1496); à la réserve près que le financement de la nouvelle édition imprimée incombait à la charge des «héritiers d'Ottaviano Scotti » en raison du décès de ce dernier au plus tard en $1503^{28}$. Mais il ne s'agit que d'indices, et de nouvelles

22. S. Giralt Soler, op. cit., p. 28, 168.

23. J. Arrizabalaga, «El libro científico en la primera imprenta castellana (1485-1520)», dans L. García BAllester éd., Historia de la ciencia y de la técnica en la Corona de Castilla. Edad Media 2, Salamanque, 2002, 4 vol., vol. II, p. 619-649, p. 630.

24. Pesenti, op. cit., p. 129.

25. Sur Tommaso Murchi, voir dans ce volume l'article de Sebastià Giralt.

26. J. A. Paniagua, «La obra médica de Arnau de Vilanova », Archivo Iberoamericano de Historia de la Medicina y Antropología Médica, 11/4, 1959, p. 351-401 à p. 377-378.

27. BAV, Rossianus 672, fo $195 \mathrm{v}^{\circ}$ : «E adì 25 zenar 1505 abrusò tutto el fontego de' Alamani o Todeschi e comenzò a 6 hore de nocte ». Sur le sens du substantif fontego, avec des allusions explicites au fontego de' Tedeschi dans le contexte vénitien, voir, sous le vocable fondaco dans sa quatrième acception, S. BATTAGLiA, Grande Dizionario della Lingua Italiana, Turin, 1961-1988, 20 vol., vol. VI, p. 122.

28. En anglais la distinction entre editor (Argilagues), printer (Locatelli) et publisher (Scotti) est très claire; il n'en va pas de même dans les langues latines où le terme «éditeur » s'appliquerait indistinctement à Argilagues et à Scotti. Afin d'éviter cette confusion assez souvent réitérée, nous avons préféré réserver pour l'occasion le terme d' 'éditeur » exclusivement à Argilagues. Aussi bien Ottaviano Scotti et ses héritiers que la majorité des publishers vénitiens, employaient un ou plusieurs imprimeurs en même temps (voir $\mathrm{HIRSCH}$, op. cit., p. 57). La date de décès d'Ottaviano Scotti a été déduite de l'année où les livres ont commencé à être signés par ses héritiers. Voir les index de l'INDEX Aureliensis. Catalogus librorum sedecimo saeculo impressorum (1962-), Aureliae Aquensis, Index Aureliensis Foundation. 
preuves seraient nécessaires pour confirmer de manière définitive cette hypothèse.

\section{Argilagues, éditeur de l'Articella et du Conciliator de Pietro d'Abano}

Argilagues fut bien conscient des nouvelles perspectives qu'offrait l'imprimerie à la récupération et la diffusion du savoir antique et médiéval. Il se sentait le protagoniste d'une nouvelle époque marquée à la fois par l'invention de Gutenberg et par la découverte récente d'une énorme quantité de manuscrits inconnus jusqu'alors. Son enthousiasme débordant, porté par l'effet multiplicateur que la mise en ouvre combinée de ces deux événements avait sur le volume de livres disponibles à son époque, est patent dans sa postface aux éditions de l'Articella.

Son profil intellectuel est caractéristique des médecins de la seconde moitié $\mathrm{du} \mathrm{Xv}^{\mathrm{e}}$ siècle, formés dans les facultés de médecine italiennes à la veille de l'irruption en leur sein du mouvement intellectuel connu sous le nom d'humanisme grec ou hellénisme. Cela veut dire que sa marque conceptuelle est le galénisme du bas Moyen Âge latin, un système fondé sur l'interprétation de la tradition médicale hippocratico-galénique par les autorités arabes, en particulier, celle d'Avicenne - raison pour laquelle on le connaît également sous le nom de galénisme avicennien. Or cela ne constitua pas un obstacle au fait qu'il fût influencé par des techniques et des valeurs propres au mouvement humaniste, bien qu'en aucune manière il ne puisse être considéré comme un humaniste à proprement parler. Comme l'a bien souligné Paul Oskar Kristeller, vers le milieu du $\mathrm{Xv}^{\mathrm{e}}$ siècle, l'influence du mouvement humaniste en Italie avait transcendé les limites des studia humanitatis et, dans une plus ou moins grande mesure, elle imprégnait tous les milieux de la culture, y compris la philosophie, les différentes sciences et la médecine ${ }^{29}$. Le profil médical d'Argilagues permet d'expliquer aussi bien les œuvres qu'il choisit d'éditer - les Articella et le Conciliator de Pietro d'Abano - que sa position dans les débats importants de son temps - position que l'on peut déduire du contenu de ses différents écrits et de ses propres annotations qu'il a introduites dans ses éditions.

Avant d'approfondir l'analyse de son profil médical, j'examinerai brièvement la signification historique de l'Articella et du Conciliator de Pietro d'Abano dans la médecine du bas Moyen Âge latin et les grands traits du travail éditorial d'Argilagues dans les deux œuvres.

\section{L'Articella}

L'Articella est une collection médicale d'écrits brefs qui soumettaient les rudiments de la médecine hippocratico-galénique à une finalité d'enseigne-

29. Voir, par exemple, P. O. KRISTELler, Renaissance thought and its sources, New York, 1979, p. 29-30. 
ment. Elle fut établie durant le XII ${ }^{\mathrm{e}}$ siècle et au début du XIII ${ }^{\mathrm{e}}$ siècle, par les médecins salernitains, lesquels également instituèrent la pratique de l'enseignement fondé sur le commentaire de ces textes. L'Articella perdura de façon étonnante dans les facultés de médecine, constituant un instrument indispensable de l'enseignement médical dans les universités du XII ${ }^{\mathrm{e}}$ siècle jusqu'au début de la décennie 1530 au moins. Avant l'avènement de l'imprimerie, elle sut très bien s'adapter aux temps nouveaux, et elle fut l'une des œuvres médicales qui eut la plus grande fortune éditoriale durant la première étape de l'imprimerie : six éditions avant la fin $d u \mathrm{Xv}^{\mathrm{e}}$ siècle et au moins dix durant les quatre premières décennies du $\mathrm{XVI}^{\mathrm{e}}$ siècle $^{30}$.

Argilagues fut l'initiateur des éditions vénitiennes de l'Articella, publiées en 1483 et 1487 . De plus, quatre éditions vénitiennes ultérieures de cette collection médicale - 1491, 1493, 1500 et 1513 -, où figure comme éditeur Gregorio da Volpe, ont un contenu identique et un format très semblable à celui des éditions préparées par Argilagues. Son travail éditorial se matérialisa, entre autres, par l'incorporation à la collection de nouvelles œuvres qui n'étaient pas incluses dans l'editio princeps de Padoue (ca 1476). En effet, aux sept écrits inclus dans cette dernière (l'Isagoge de Joannitius, le De pulsibus de Philaret, le De urinis de Théophile, les Aphorismi, les Prognostica et le De regimine acutorum morborum hippocratiques accompagnés des commentaires de Galien, ainsi que l'Ars medica ou le Tegni de Galien avec le commentaire d'Ali-ibn-Ridwan), Argilagues ajouta quatre œuvres supplémentaires (Epidemiae cum commentis Johannis Alexandrini, De natura fetus, De lege et Iusiurandum), appartenant toutes au Corpus Hippocraticum et imprimées pour la première fois à cette occasion ${ }^{31}$. Son initiative rend patent le rôle que joua aussi l'éditeur de la première imprimerie : celui consistant à offrir de nouveaux horizons intellectuels. De plus, il introduisit l'opuscule De divisione librorum Galieni de Gentile da Foligno et trois tables (tabulae) - qu'il élabora sans doute lui-même - relatives aux œuvres hippocratiques et galéniques éditées (une pour les Aphorismi; une seconde à la fois pour les Pronostica, le De regimine acutorum morborum et les Epidemiae; et une troisième pour le Tegni), dont la finalité était de faciliter l'accès à l'information abondante et variée qu'elles contenaient.

\section{Le Conciliator de Pietro d'Abano}

L'autre production éditoriale de Argilagues fut le Conciliator differentiarum medicorum et philosophorum de Pietro d'Abano (1250-ca 1315), médecin, professeur à l'Université de Padoue et éminent représentant de ce qu'on

30. Sur l'Articella et sa diffusion dans la première imprimerie, voir la monographie de ArrizaBALAGA citée à la note 5, et la bibliographie qui s'y trouve.

31. Stillwell, op. cit., p. 129-130, 209 (\# 411, 414, 415, 659). 
appelle l' «aristotélisme padouan ${ }^{32}$. Organisée autour de 210 « différences » (differentiae) qui suivent la structure de la quaestio scolastique, cette œuvre passe en revue l'ensemble du savoir médical hippocratico-galénique, en recherchant toujours l'harmonisation des opinions entre médecins et philosophes de la nature sur les points qui font l'objet des principales controverses entre les deux groupes professionnels.

Le Conciliator aussi bénéficia d'une notable fortune éditoriale, de 1472 jusqu'assez tard dans la seconde moitié du $\mathrm{XVI}^{\mathrm{e}}$ siècle : cinq éditions imprimées avant la fin $\mathrm{du} \mathrm{XV}^{\mathrm{e}}$ siècle et au moins six jusqu'en 1565. Comme Argilagues le reconnaît lui-même dans son prologue, cette œuvre avait déjà été imprimée à deux occasions (Mantoue 1472 et Venise 1476) avant que son édition ne parût à Venise en février 1483, à peine deux mois avant la première édition, imprimée également à Venise, de son Articella. L'édition d'Argilagues du Conciliator fut réimprimée à deux reprises avant la fin $\mathrm{du} \mathrm{Xv}^{\mathrm{e}}$ siècle (Pavie 1490 et Venise 1496).

Le zèle éditorial d'Argilagues fut une nouvelle fois patent dans son édition du Conciliator. En effet, dans les éditions de 1483 et 1490 il avait inclus à la fin de l'œuvre de Pietro d'Abano l'annexe de la «différence » $37^{33}$ et celle de la «différence » $65^{34}$. Son édition de 1496 allait incorporer l'une et l'autre de ces annexes au texte des differentiae correspondantes et allait ajouter une autre annexe à la « différence » 92, accueillie aux folios $264 \mathrm{r}^{\circ}-265 \mathrm{r}^{\circ}$, à la fin du Tractatus de venenis - une autre ouvre de Pietro d'Abano qui accompagna toutes les éditions incunables du Conciliator, sauf celle de 1483. Il convient aussi de signaler que la table des differentiae ou quaesita, qui se trouve dans les deux premières éditions imprimées du Conciliator, immédiatement après le prologue, fut déplacée à la fin de l'œuvre dans les trois éditions dont se chargea Argilagues : à la suite des annexes, dans l'édition de 1483 et $1490^{35}$ et immédiatement avant l'annexe, dans l'édition de 1496 $\left(\mathrm{f}^{\mathrm{o}} 255 \mathrm{r}^{\mathrm{0}}-256 \mathrm{v}^{\circ}\right)$.

\section{Tradition médiévale et culture humaniste dans les activités éditoriales de Francesc Argilagues}

Je vais maintenant m'attacher à certaines particularités de l'Articella d'Argilagues qui permettent de mieux illustrer le profil intellectuel de ce médecin universitaire de la fin du $\mathrm{Xv}^{\mathrm{e}}$ siècle. En premier lieu, ce n'est pas

32. Sur Pietro d'Abano et son œuvre, voir F. ALEssio, «Filosofia e scienza. Pietro d'Abano », Storia della cultura veneta. 2. Il Trecento, Vicence, 1976, p. 171-206; E. PAScheTto, Pietro d'Abano medico e filosofo, Florence, 1985 ; L. Olviveri, Pietro d'Abano e il pensiero neolatino, Padoue, 1988 ; et les articles recueillis dans Medioevo. Rivista di storia della filosofia medievale, 11, 1985 (numéro monographique consacré à cette figure).

33. Pietro d'Abano, Conciliator, Venise, Johann Herbort, 1483, signatures. gg2v-gg3v; Pavie, Gabriele Grassi, 1490, signatures. M2v-M3v.

34. Pietro d’Abano, Conciliator, Venise, Johann Herbort, 1483, signatures. gg3v-gg4r ; Pavia, Gabriele Grassi, 1490, signatures. M3v-M4r.

35. Pietro d'Abano, Conciliator, Venise, Johann Herbort, 1483, signatures. gg4r-gg5v ; Pavia, Gabriele Grassi, 1490, signatures. M4r-M5v. 
un hasard si dans sa note introductive de présentation et de justification des écrits compris dans son Articella, en se référant au livre hippocratique des «Pronostics » avec les commentaires de Galien, il fait une petite digression - en réalité, une quaestio scolastique - à propos du calcul des «jours critiques » dans la fièvre (febris) post partum, où il résout une apparente divergence entre Galien et Avicenne sur cette question, en conciliant les opinions des deux auteurs ${ }^{36}$; il faut en effet tenir compte aussi bien du rattachement déjà mentionné d'Argilagues au galénisme avicennien que du statut d'Avicenne perçu comme le principal ennemi à combattre dans le programme de réforme de la médecine promue par les hellénistes.

D'autre part, la position d'ouverte confrontation d'Argilagues avec les éditeurs héllénistes apparaît de façon manifeste. Dans un paragraphe de sa note introductive à l'Articella, très significatif à cet égard, il informe le lecteur de certaines particularités de son édition du De regimine acutorum morborum d'Hippocrate :

En septième lieu, on trouve le livre Sur la diète dans les maladies aiguës divisé en quatre parties, dont trois furent éditées dans les précédentes années, mais non la quatrième en raison de la difficulté qu'elle présente. Pour cette dernière il $\mathrm{y}$ a seulement une traduction. Si dans cette dernière partie apparaissent écrits de façon incorrecte en caractères latins certains mots grecs, qui feront peut-être rire quelque expert en grec, il n'y a pas lieu de critiquer cela, puisque les versions de ces mots sont fidèles et exactes. Aucun des manuscrits dont j'ai disposé lorsque j'ai fait la correction ne diverge quant aux dits mots, même s'ils apparaissent fréquemment écrits de manière différente dans le commentaire et dans le texte. Du moment que l'on connaît l'essence de la chose, il ne faut pas se préoccuper des mots ; c'était le désir de Galien d'arriver à apprendre et à enseigner sans eux. Ainsi, on pourrait alléguer contre un grand nombre de personnes ce passage de la seconde partie des Aphorismes, dans le commentaire 22: «Je veux contourner les opinions des nouveaux médecins qui ne cessent de bavarder sur les noms, en croyant se référer aux choses auxquels ceux-ci correspondent. » Nous pourrions également recourir au livre III du Tegni [de Galien], vers la fin, où il dit : « Il est aussi possible de ne pas nommer les causes comme le font nombre de ces sophistes qui négligent la recherche visant à découvrir l'immense diversité des choses et limitent leur vie à des questions de noms. » Averroès, pour sa part, dit que la préoccupation d'Aristote pour le nom fut rare. Les versions latines doivent suffire au lecteur, car le latin ne doit pas être considéré comme inférieur en dignité et en excellence au grec. Dans le prologue aux Questions tusculanes, Cicéron dit : «J'ai toujours pensé que nos aînés étaient en eux-mêmes plus intelligents en tout que les Grecs ou qu'ils ont amélioré ce qu'ils ont pris à ces derniers »; bien que Priscien et les autres pensent le contraire ${ }^{37}$.

36. ARTiCelLa, Venise, Hermann Liechtenstein, 1483, $\mathrm{f}^{\circ} 1 \mathrm{v}^{\circ}$. Texte latin et traduction espagnole reproduits dans Arrizabalaga, García Ballester et Gil Aristu, op. cit., p. 39-40, 43-44.

37. ARticElLA, op. cit., $\mathrm{f}^{\mathrm{o}} 1 \mathrm{v}^{\circ}$. Texte latin et traduction espagnole reproduits dans Arrizabalaga, García Ballester et Gil Aristu, op. cit., p. 40-41, 44-45. 
Il s'agit d'un témoignage de première main sur l'une des polémiques les plus significatives qui eurent lieu au sein des cercles intellectuels italiens pendant les dernières décennies du $\mathrm{Xv}^{\mathrm{e}}$ siècle et le début $\mathrm{du} \mathrm{XVI}^{\mathrm{e}}$, celle qui opposa l'establishment médical académique aux premiers médecins hellénistes : les vétérans, qui défendaient la valeur et la légitimité de la tradition culturelle du bas Moyen Âge comme fondement de la médecine ; et les jeunes, qui prônaient le retour à la prisca medicina grecque comme unique moyen d'effectuer la réforme du savoir médical qu'ils jugeaient indispensable. Par conséquent, les uns défendaient le caractère idoine et autosuffisant du latin comme langue du savoir médical, tandis que les autres considéraient le grec comme un instrument inévitable de la «renaissance » médicale ${ }^{38}$.

Le mouvement médical helléniste, dont les premiers noyaux se cristallisèrent dans des figures comme celles de Nicolò Leoniceno (1428-1524) et Giorgio Valla (1447-1500), se diffusa rapidement, d'abord en Italie, puis dans le reste de l'Europe, atteignant sa plénitude tout au long du XVI ${ }^{\mathrm{e}}$ siècle. Parmi ses protagonistes il convient de citer aussi Lorenzo Lorenzano (1450-ca 1502), Giovanni Manardi (1462-ca 1536), Jean de la Ruelle (ca 1479-1537), Wilhelm Kop (1460-ca 1532), Johann Guinther von Andernach (1505-1574) et Thomas Linacre (ca 1460-1524). Bien évidemment, l'une des activités les plus remar-

38. Sur l'humanisme et, en général, sur la culture des élites italiennes et européennes de la fin du $\mathrm{XV}^{\mathrm{e}}$ siècle et du début du XVI $\mathrm{X}^{\mathrm{e}}$ siècle, on peut voir, entre autres, R. R. BolgaR, The Classical Heritage and its beneficiaries, Cambridge, 1954 ; L. D. Reynolds et N. G. WiLson, Scribes and scholars. A guide to the transmission of Greek and Latin literature, $3^{\mathrm{e}}$ éd., Oxford, 1991 ; KRISTELLER, op. cit.; J. STEPHENS, The Italian renaissance. The origins of intellectual and artistic change before the Reformation, Londres/New York, 1990 ; A. GoOdman et A. MACKAY éd., The impact of humanism on Western Europe. Londres/New York, 1990 ; J. Hankins, Plato in the Italian Renaissance, $2^{\mathrm{e}}$ impr., Leyde, 1991, 2 vol.; W. RÜEGG, "Epilogue : the Rise of Humanism », dans H. DE RIDDER-SYMOENS éd., A History of the University in Europe. Volume 1 : Universities in the Middle Ages, Cambridge, 1992, p. 442-468; F. Rico, El sueño del humanismo. (De Petrarca a Erasmo), Madrid, 1993. Sur la culture médicale et philosophico-naturelle universitaire pendant cette période, on peut voir, entre autres, R. DuRLING, «A chronological census of Renaissance editions and translations of Galen », Journal of the Warburg and Courtauld Institutes, 24, 1961, p. 230-305 ; F. Maddison, M. Pelling et Ch. Webster, éd., Linacre Studies. Essays on the Life and Work of Thomas Linacre, ca 1460-1524, Oxford, 1977 ; J. J. BYLEBYL, "The School of Padua: humanistic medicine in the sixteenth century », dans Ch. WebsTER éd., Health, medicine and mortality in the sixteenth century, Cambridge, 1979, p. 335-370; R. K. FRENCH, "Berengario da Carpi and the use of commentary in anatomical teaching », dans A. Wear, R. K. French et I. M. Lonie éd., The medical renaissance of the sixteenth century, Cambridge, 1985, p. 42-74, 296-298 ; R. K. FRENCH, «Pliny and Renaissance medicine », dans R. K. French et F. Greenaway, éd., Science in the Early Roman Empire : Pliny the Elder, his Sources and Influence, Londres/Sidney, 1986, p. 252-281 ; N. G. SIRAISI, Avicenna in renaissance Italy. The Canon and medical teaching in Italian universities after 1500, Princeton, 1987 ; V. Nutton, John Caius and the manuscripts of Galen, Cambridge, 1987 ; D. MugnaI-CARRARA, La biblioteca di Nicolò Leoniceno. Tra Aristotele e Galeno : cultura e libri di un medico umanista, Florence, 1991 ; A. GRAFTON, Defenders of the text. The traditions of scholarship in an age of science, 1450-1800, Cambridge, Mass., 1991 ; J. Arrizabalaga, J. Henderson et R. K. French, The Great Pox. The French Disease in Renaissance Europe, New Haven/Londres, 1997; V. NutTon, «The rise of medical humanism : Ferrara, 1464-1555 », Renaissance Studies, 11/1, 1997, p. 2-19. 
quables des médecins hellénistes fut l'édition et la traduction des écrits d'autorités médicales grecques, aussi bien antiques que byzantines. Leurs nouvelles traductions gréco-latines des ouvres d'Hippocrate, de Galien et d'autres commencèrent à circuler, manuscrites, en Italie à partir de 1480, mais - ce qui donne bien la mesure de la résistance considérable opposée par l'establishment académique aux propositions de réforme - la grande majorité d'entre elles ne devaient pas être imprimées avant $1500^{39}$. (Il ne faut pas oublier qu'Alde Manuce, paradigme de l'imprimerie humaniste, commença ses activités éditoriales à Venise en 1494, et que c'est seulement à partir de 1497 qu'il se lança dans la publication de nouvelles éditions en latin et en grec des grands maîtres de la médecine et de la philosophie antiques.)

Le passage cité plus haut met en évidence l'alignement résolu d'Argilagues sur les positions des partisans de la tradition médicale latine du bas Moyen Âge. En effet, il défendait l'excellence des versions latines médiévales comprises dans son Articella, en même temps qu'il mettait en cause la valeur des nouvelles traductions des hellénistes, qui commençaient alors à circuler sous forme manuscrite, entrant ainsi directement en concurrence avec les premières. Argilagues critiquait la méthodologie des traducteurs hellénistes, invoquant le fait que les préoccupations philologiques (de nominibus) de ces derniers les détournaient de ce qui aurait dû préoccuper les médecins et les philosophes de la nature (de rebus). L'exaspération de la confrontation entre les deux courants intellectuels est patente dans le ton virulent et manifestement méprisant des critiques d'Argilagues : il comparait les hellénistes aux «nouveaux médecins» (medicorum novorum) et aux sophistes que Galien accusait de s'attarder sans fin dans des disputes nominalistes en négligeant la recherche sur la nature et la diversité des choses.

Argilagues, qui justifiait l'usage en médecine de la langue latine, en affirmant que celle-ci possédait une dignité et une excellence qui n'étaient pas inférieures à la langue grecque, s'identifiait sans réserve à la tradition latine du bas Moyen Âge, aussi bien dans le champ médical que dans celui de la philosophie naturelle. Par conséquent, il revendiquait d'une manière orgueilleuse et sans tarir d'éloges l'immense œuvre de traduction de Gérard de Crémone (1114-1187), l'une des figures les plus représentatives de cette tradition et, sans doute, le représentant le plus remarquable du mouvement des traducteurs qui mirent d'arabe en latin le legs constitué par les œuvres de médecine et de philosophie naturelle de l'Antiquité et du monde arabe dans la Tolède des $\mathrm{XII}^{\mathrm{e}}$ et XIII ${ }^{\mathrm{e}}$ siècles ${ }^{40}$.

39. Durling, op. cit. ; R. J. DurLing, « Corrigenda et addenda to Diels' Galenica », Traditio, 23, 1967, p. 461-476 ; 37, 1981, p. 373-381 ; StILlwell, op. cit., p. 113-117, 125-131 ; NuTTON, op. cit., p. 19-49.

40. ARtiCELLA, op. cit., fo $1 \mathrm{v}^{0}$ : « ...Gérard de Cremone, homme très illustre qui traduit de l'arabe au latin 75 œuvres aussi de dialectique et de philosophie comme de mathématiques, en plus de 21 œuvres de médecine. Si l'occasion était propice, j'énumérerais ici ces travaux en son honneur ». Texte latin et traduction espagnole reproduits dans ArRIZABALAGA, GarCía BALLESTER et GIL ARISTU, op. cit., p. 41, 45. 
Comme l'on pouvait s'y attendre, la quasi-totalité des œuvres comprises dans l'Articella d'Argilagues procédaient de la tradition du bas Moyen Âge, dans des traductions latines effectuées à la fois à partir de l'arabe et du grec. Si l'on prend comme échantillon les huit œuvres qui appartiennent à Hippocrate et Galien recueillies dans cette Articella, six procédaient de versions arabo-latines de Constantin l'Africain (seconde moitié du XI ${ }^{\mathrm{e}} \mathrm{s}$.) et de Gérard de Crémone (seconde moitié du XII ${ }^{\mathrm{e}}$ s.) ; les deux autres, De natura fetus et Iusiurandum, correspondaient respectivement à des traductions gréco-latines de Barthélemy de Messine (seconde moitié du XIII ${ }^{\mathrm{e}}$ s.) et de Nicolò Perotti (1429/1430-1480).

Or le rattachement d'Argilagues à la tradition médicale du bas Moyen Âge n'a pas constitué un obstacle au fait que son travail ait répondu aux meilleures exigences de la technique éditoriale contemporaine. La pénétration et l'influence exercées tout au long $\mathrm{du} \mathrm{Xv}^{\mathrm{e}}$ siècle par le courant humaniste au sein des facultés de médecine italiennes devait nécessairement se refléter dans la tâche éditoriale d'Argilagues et d'autres médecins européens de sa génération intellectuelle. De fait, Argilagues dénonçait ouvertement l'énorme quantité d'erreurs et de coquilles contenues dans les traductions (imprimées et manuscrites) alors en circulation des œuvres des "médecins antiques ». Il estimait que les défauts de la majorité des passages étaient tels que l'on ne pouvait en tirer «aucun sens et aucune opinion». Tout cela lui paraissait particulièrement grave dans le cas d'Hippocrate, dont il revendiquait ouvertement la figure comme un «homme de nature supérieure», "grand bienfaiteur du genre humain », qui « rendit la lumière à la médecine, qui s'était depuis longtemps fourvoyée ${ }^{41}$. Et bien qu'il critiquât comme quelque chose de fréquent chez les éditeurs scientifiques, le manque de «labeur et de soin dans la correction des livres», qu'il rendait responsable des constantes « invraisemblances et extravagances » qui existaient dans les œuvres imprimées, il réservait l'attaque la plus forte aux imprimeurs, dont il disait qu' « ils ont l'habitude d'altérer et de changer presque toujours tout ce qu'ils reçoivent corrigé ${ }^{42}$. Une critique des imprimeurs qui révèle la tension entre le travail d'éditeur et celui d'imprimeur, provenant d'une différence d'intérêts et de sensibilités de l'un et de l'autre dans le processus d'élaboration, de distribution et de vente du livre imprimé dans le contexte d'un marché terriblement compétitif. Cependant, le fait de se démarquer de ceux qui offraient des produits intellectuels imprimés de faible qualité peut être aussi interprété comme une habile défense de la tradition médicale latine du bas Moyen Âge face aux attaques hellénistes contre des cibles trop faciles.

(Traduit de l'espagnol par Nicolas WeILL-PAROT)

Jon Arrizabalaga, Dpto. de Historia de la Ciencia. CSIC - Institución Milà i Fontanals, Egipcíaques, 15, E-08001 Barcelona

41. ARticella, op. cit., $\mathrm{f}^{\mathrm{o}} 211 \mathrm{v}^{\mathrm{o}}$. Texte latin et traduction espagnole reproduits dans Arrizabalaga, García Ballester et Gil Aristu, op. cit., p. 29-30, 33.

42. ARTICELLA, op. cit., fo $211 \mathrm{v}^{\circ}$. Texte latin et traduction espagnole reproduits dans Arrizabalaga, García Ballester et Gil Aristu, op. cit., p. 30-31, 33-34. 
De la copie à l'édition : Francesc Argilagues et les manuscrits médicaux aux premiers temps de l'imprimerie (fin $\mathrm{XV}^{\mathrm{e}}$-début $\mathrm{XVI}^{\mathrm{e}}$ siècle)

La biographie de Francesc Argilagues (fl. Sienne, ca 1470-Venise, 1508), médecin espagnol établi en Italie après des études universitaires à Sienne et Pise, illustre dans le domaine de la médecine universitaire la période de transition entre la copie de manuscrits et les premières éditions imprimées; et elle reflète les débuts de la fonction d'éditeur scientifique, avec une attention particulière aux questions de nature technique ou idéologique auxquelles furent confrontés ceux qui s'adonnèrent à cette nouvelle occupation surgie de l'invention de Gutenberg.

\section{From Copying to Editing : Francesc Argilagues and the Medical Manuscripts in the Times of the Early Printing Press, late 15th-early 16th century}

The activities of Francesc Argilagues (fl. Siena, ca 1470-Venice, 1508), a Spanish medical practitioner who settled in Italy after having studied at the universities of Siena and Pisa, illustrate in the area of university medicine, the transitional period between copying medical manuscripts and editing them for the early printing press ; and reflect the beginnings of the scientific editor's job with particular attention to the technical and ideological questions faced by those scholars who were involved in this new occupation emerged as a result of Gutenberg's invention. 
